\title{
Constitutive relations for the isotropic deformation of frictionless packings of polydisperse spheres
}

\author{
Fatih Göncü ${ }^{\mathrm{a}, \mathrm{b}}$, Orencio Durán ${ }^{\mathrm{a}, \mathrm{c}}$, Stefan Luding ${ }^{\mathrm{a}, \mathrm{b}}$ \\ ${ }^{a}$ Multi Scale Mechanics (MSM), Faculty of Engineering Technology, University of Twente, P.O. Box 217, 7500 AE \\ Enschede, The Netherlands \\ b NanoStructured Materials (NSM), ChemTech, Delft University of Technology, Delft, The Netherlands \\ ${ }^{\mathrm{c}}$ PMMH, UMR7636 (CNRS), ESPCI Univ. P6-P7, 10 Rue Vauquelin, 75005 Paris, France
}

\begin{abstract}
The isotropic compression of polydisperse packings of frictionless spheres is modeled with the discrete element method (DEM). The evolution of coordination number, fraction of rattlers, isotropic fabric, and pressure (isotropic stress) is reported as function of volume fraction for different system parameters. The power law relationship, with power $\approx 1 / 2$, between coordination number and volume fraction is confirmed in the jammed state for a broad range of volume fractions and for different (moderate) polydispersities. The polydispersity in the packing causes a shift of the critical volume fraction, i.e., more heterogeneous packings jam at higher volume fractions. Close to jamming, the coordination number and the jamming volume fraction itself depend on both history and rate. At larger densities, neither the deformation history nor the loading rate have a significant effect on the evolution of the coordination number.

Concerning the fabric tensor, comparing our DEM results to theoretical predictions, good agreement for different polydispersities is observed. An analytical expression for the pressure as function of isotropic (volumetric) strain is proposed for polydisperse packings, based on the assumption of uniform deformation. We note that, besides the implicit proportionality to contact number density (or fabric), no single power-law is evidenced in the relation for the pressure. However, starting from zero pressure at the jamming point, a linear term with a quadratic correction describes the stress evolution rather well for a broad range of densities and for various polydispersities. Finally, an incremental evolution equation is proposed for both fabric and stress, as function of isotropic strain, and involving the coordination number and the fraction of rattlers, as starting point for further studies involving anisotropic deformations.
\end{abstract}




\section{Résumé}

Lois de comportement pour déformations isotrope d'assemblage de sphères polydisperses sans frottement

La compression isotrope d'assemblages polydisperses de sphères sans frottement est modélisée par une méthode aux éléments discrets (DEM). L'évolution du nombre de coordination, de la fraction de "rattlers" (les particules instables, sans contactes), de la texture isotrope et de la pression (contrainte isotrope) est étudiée en fonction de la fraction volumique pour différentes valeurs des paramètres du système. Une relation en loi puissance, avec un exposé proche de 0.5 , entre le nombre de coordination et la fraction volumique est confirmée en régime de blocage pour une large gamme de fractions volumiques et pour différentes polydispersités. La polydispersité de l'assemblage induit un décalage de la fraction volumique critique, c'est-à-dire que les assemblages plus hétérogènes se bloquent à des fractions volumiques plus élevées. Au voisinage du jamming, le nombre de coordination et la fraction volumique de blocage dépendent à la fois de l'histoire et de la vitesse de chargement. A des densités plus élevées, ni l'histoire des déformations et ni la vitesse de chargement ont un effet significatif sur l'évolution du nombre de coordination.

En ce qui concerne le tenseur de texture, la comparaison de nos résultats DEM avec les prédictions théoriques est satisfaisante pour différentes polydispersités. Une expression analytique de la pression en fonction des déformations volumiques est proposée pour différents assemblages polydisperses, fondée sur une hypothèse de déformation uniforme. On notera que, outre la proportionnalité implicite vis-à-vis de la densité de nombre de contacts, aucune loi puissance ne peut être mise en évidence dans la relation donnant la pression. Cependant, partant d'une pression nulle au point de blocage (jamming), un terme linéaire peut décrire, avec une correction quadratique, l'évolution de la contrainte de manière satisfaisante, pour une large gamme de densités et pour diverses polydispersités. Finalement, une équation d'évolution incrémentale est proposée à la fois pour la texture et la contrainte, en fonction de la déformation volumique, et impliquant le nombre de coordination et la fraction de rattlers. Elle constitue un point de départ pour de futurs travaux en relation avec les déformations anisotropes.

Key words: polydisperse, frictionless granular materials ; isotropic compression; constitutive models ; rattlers

Mots-clés : matériaux granulaires polydisperses sans frottement; compression isotrope; lois de comportement; particules instables (rattlers)

\section{Introduction}

Dense granular materials show peculiar mechanical properties quite different from classical fluids or solids $[1,2]$. This is true not only for realistic contact forces involving friction and adhesion [3, 4], but already in the frictionless case. Describing granular matter with continuum models is difficult due to their inherent discrete structure and since the origin of their behavior is far from understood $[4,5,6,7,8]$.

The transition from liquid to solid phases in disordered systems is generally investigated in the context of jamming [6, 7, 9]. Liu and Nagel [5] have suggested that this concept can be applied to different materials in a single framework using a jamming phase diagram with temperature, shear stress, and volume fraction as control parameters. (The volume fraction is the ratio of solid volume to total volume.) For athermal systems like granular materials jamming, i.e., the transition from fluid-like to solid-like behavior, is then essentially determined by the volume fraction and the shear stress $[10,11,12,13]$. Particularly, if a granular packing is subject to isotropic compression the shear stress is practically zero and the only control parameter is the volume fraction, or equivalently the density (which is the product of

Email addresses: f.goncu@utwente.nl (Fatih Göncü), s.luding@utwente.nl (Stefan Luding). 
volume fraction and material density). Recent numerical and experimental studies with disk and sphere assemblies were performed to identify the critical value at which jamming first occurs $[6,14,15,16]$. For monodisperse systems it corresponds approximately to the random close packing $[9,15,16]$. Other quantities such as coordination number and pressure were reported to evolve as power laws of the volume fraction in a small interval above the jamming density $[6,7,15]$, resembling a phase transition and critical phenomena $[1,2,5,7,15]$.

Another issue is predicting the mechanical properties of granular materials, which are controlled by the internal structure of the assembly of grains - where the internal structure itself depends on the history of the sample. Although, particles are much smaller than the packing, the presence of discrete force chains in the contact network can lead to long range correlations and thus precludes a straightforward continuum description. Fluctuations of quantities like stress are extreme on the particle scale, i.e., much larger than the mean values, and only over rather large representative volumina these fluctuations decay.

The fabric tensor is commonly used as first harmonic approximation to quantify the structure in disordered systems with an average and a deviatoric (anisotropic) contact density [17, 18]. Numerical studies of the fabric tensor under isotropic deformation of systems with disks, for different polydispersities, have been realized $[17,19]$ and at least the contact number density could be related to the first three moments of the size-distribution for isotropic situations. Advanced constitutive models within the framework of continuum mechanics employ various definitions of the fabric tensor as a non-classical field. For example, elasto-plasticity and hypoplasticity $[20,21]$ were generalized to include more general structure field variables, however, accurate modelling of the effect of structure on the anisotropy of granular materials remains a challenge.

The goal of this study is to test the validity of the power law for the coordination number in polydisperse packings of frictionless spheres also at relatively high volume fractions above jamming and to provide incremental evolution equations for fabric and stress under isotropic deformation. For this, we perform DEM simulations, as introduced in section 2, with packings of different polydispersities, number of particles and loading rates. In Secs. 3 and 4, we analyze numerically the evolution of the coordination number and of the (isotropic) trace of fabric as function of volume fraction and compare the result with theoretical predictions in Refs. [17, 22]. In section 5, based on a theory derived in Ref. [22], we present an analytical expression for the pressure as function of the volume fraction, resulting in an incremental evolution equation for isotropic structure (fabric) and stress.

\section{Simulation method}

The Discrete Element Method (DEM) [3, 4, 23] allows us to enclose frictionless particles in a cubic volume with periodic boundary conditions. A linear viscoelastic contact model determines the particle contact forces in the normal direction. In order to reduce dynamical effects and shorten relaxation times an artificial viscous background dissipation proportional to the particle velocity is added, resembling the damping due to a background medium. In all simulations gravity is neglected, so that the applied deformations can be assumed isotropic.

\subsection{Simulation Parameters}

Typical values of the simulation parameters are: system size $N=1000,5000$, or 10000 particles with average radius $\langle r\rangle=1[\mathrm{~mm}]$, density $\rho=2000\left[\mathrm{~kg} / \mathrm{m}^{3}\right]$, elastic stiffness $k_{n}=10^{8}\left[\mathrm{~kg} / \mathrm{s}^{2}\right]$, particle damping coefficient $\gamma=1[\mathrm{~kg} / \mathrm{s}]$, background dissipation $\gamma_{b}=0.1[\mathrm{~kg} / \mathrm{s}]$ (see Ref. [4] for a discussion of these artificial units, which can be re-scaled due to the simplicity of the contact model). Since the 
particle size distribution is polydisperse, the contact time depends on the radius of the particles. For example, $t_{c}=0.31[\mu \mathrm{s}]$ is the duration of a contact between the smallest and the biggest particles, with the polydispersity parameter $w=r_{\max } / r_{\min }=3$ as defined below. The contact time between two average particles with $r /\langle r\rangle=1$, is $t_{c}=0.64\left[\mu \mathrm{s}\right.$ ] and their mutual coefficient of restitution is $r=0.92$. Because $t_{c}$ is stiffness dependent and can be scaled arbitrarily [4], we do not consider it as an important simulation parameter (as long as the deformation is performed slow, i.e., quasi-statically). Increasing stiffness leads to smaller $t_{c}$, i.e., the system has a shorter response time, but has otherwise no effect on the quasi-static results presented in this study.

In order to quantify the volume fraction rate of change during isotropic deformation, the relative loading rate for packings undergoing the same deformation is defined as $D=T_{\text {ref }} / T_{\text {sim }}$, where $T_{\text {ref }}=1000[\mu \mathrm{s}]$ is the duration of the fastest simulation. Values of $D$ used for simulations are $10^{-3}, 10^{-2}, 10^{-1}$ and 1 .

A typical deformation is applied in a strain-controlled manner to the system boundaries (periodic "walls"), with a cosine-shape in order to avoid shocks. In a few cases, other strain functions such as pressure-controlled "wall" displacement and uniform strain field deformation were tested. In the latter case, the particle displacements are determined such that the instantaneous strain field is uniform inside the packing, but relaxation is allowed due to the interactions. We observe that there are no strong differences in the simulation results obtained from different methods as long as the deformation rates are small. (Therefore we do not discuss the actual strain rate, but refer to the scaled (relative) inverse period of deformation $D=T_{\text {ref }} / T_{\text {sim }}$ as dimensionless rate.)

\subsection{Polydispersity}

The polydispersity of the particles can be quantified by the width $w=r_{\max } / r_{\min }$ of the uniform distribution:

$$
f(r)=\frac{w+1}{2(w-1)\langle r\rangle} \Theta\left(\frac{2 w\langle r\rangle}{w+1}-r\right) \Theta\left(r-\frac{2\langle r\rangle}{w+1}\right),
$$

with the step function $\Theta(x \geq 0)=1$ and $\Theta(x<0)=0$. The dimensionless moments of the size distribution can be expressed as functions of $w$ :

$$
\hat{r}_{k}:=\frac{\left\langle r^{k}\right\rangle}{\langle r\rangle^{k}}=\frac{2^{k}}{(k+1)(w+1)^{k}} \sum_{i=0}^{k} w^{i},
$$

with the first two moments $\hat{r}_{1}=1$, and $\hat{r}_{2}=\frac{4}{3} \frac{1+w+w^{2}}{(w+1)^{2}}$. Typical values of $w$ are 1,2 and 3 , where $w=1$ corresponds to a monodisperse packing. A few simulations with larger $w \leq 8$ were also performed. Simulations with other size distribution functions and a theoretical analysis of polydisperse packings will be published elsewhere [22].

\subsection{Preparation and test procedure}

The initial packing is obtained by compressing a (fully) random granular "gas" up to a volume fraction close to jamming and letting it relax. Figure 2 shows the initial configuration of the particles, the granular gas state, before, and the granular fluid state, after first relaxation at an initial volume fraction below jamming $\nu_{\mathrm{i}}=0.64$. From the granular fluid, below jamming, the system is slowly compressed and the evolution of the kinetic and potential energies is displayed during relaxation and compression. The packings are isotropically compressed by moving simultaneously inwards the (fictive, periodic) boundaries 


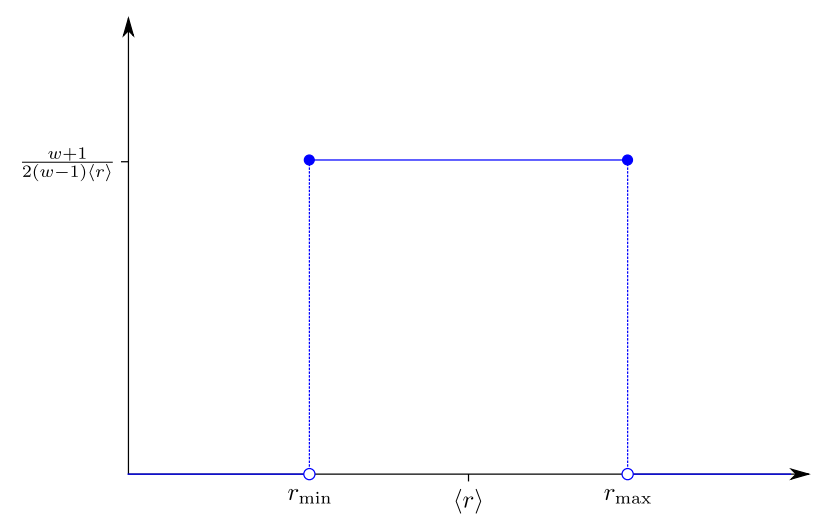

Figure 1. Probability density function of the uniform distribution.

of the simulation domain, see Figs. 2(b)-(d). After maximal compression to $\nu_{\max }=0.75$, the process is reversed until the initial volume fraction $\nu_{\mathrm{i}}$ is recovered.

Besides (artificial) contacts at the initial state (which disappear immediately due to the high repulsive forces involved), contacts are closed permanently only above the jamming volume fraction. The potential energy is an indicator of the overlaps of the particles. However, since the compression is rather fast, one can observe considerable potential energy due to collisions in the fluid-like state, at densities $\nu_{\mathrm{i}}<\nu<\nu_{\mathrm{j}}$, with jamming volume fraction $\nu_{\mathrm{j}}$. From Fig. 2(f), in the loading or un-loading state, one observes that the kinetic energy is smaller than the potential energy at the higher densities. In the (isotropic) jammed, solid state, the potential energy is considerably larger than the kinetic energy, whereas in the fluid-like state referred to above it is significantly smaller. This is a rough indicator of the jammed regime, however, not really an objective criterion due to the dynamic loading and un-loading. Close to the maximal volume fraction, due to our co-sinusoidal loading procedure, the kinetic energy drops exponentially over about two orders of magnitude between times $t=480 \mu \mathrm{s}$ and $\sim 580 \mu \mathrm{s}$. For larger times, the rate of change increases so that the kinetic energy increases again, showing jumps whenever the packing re-arranges.

Around time $t=850 \mu \mathrm{s}$, the volume fraction drops below the un-loading jamming value and the kinetic energy becomes larger than the potential energy. Also in this fluid-like high-density granular gas, the kinetic energy drops approximately exponentially due to collisional cooling, however, with a different rate as before in the high density, slow deformation regime.

\section{Evolution of the coordination number}

In theory, the jamming transition occurs at the isostatic point $[7,15,24]$. In an isostatic packing of frictionless particles, the coordination number, i.e., the average number of contacts per particle, is $C=2 D$ where $D$ is the dimensionality of the system. One can expect smaller coordination numbers when tangential elastic forces are involved, however, even in simulations and experiments with very small tangential forces, the reported values of $C$ are consistently below $2 D$. This is due to the definition of an isostatic packing, which excludes all particles that do not belong to the force network, i.e., ideally, particles with exactly zero contacts are excluded. Nevertheless, in addition to the particles with zero contacts, there may be particles having a finite number of contacts for some short time, which do not contribute to the mechanical stability of the packing. The contacts of these rattlers are transient because the repulsive contact forces push them away from the mechanically stable backbone. Thus, if the packing were allowed to relax after every deformation step, or be deformed very slowly, these particles would lose 


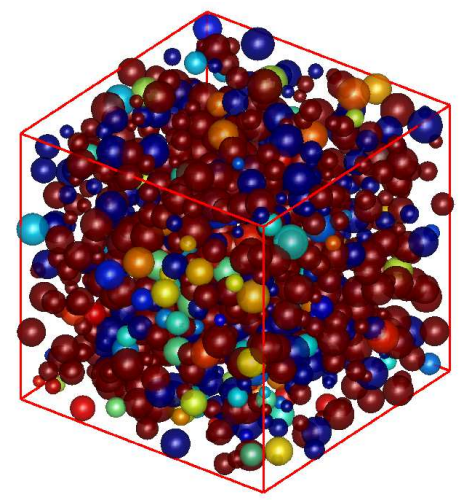

(a)

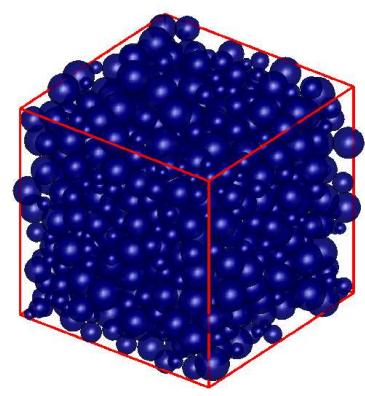

(c)

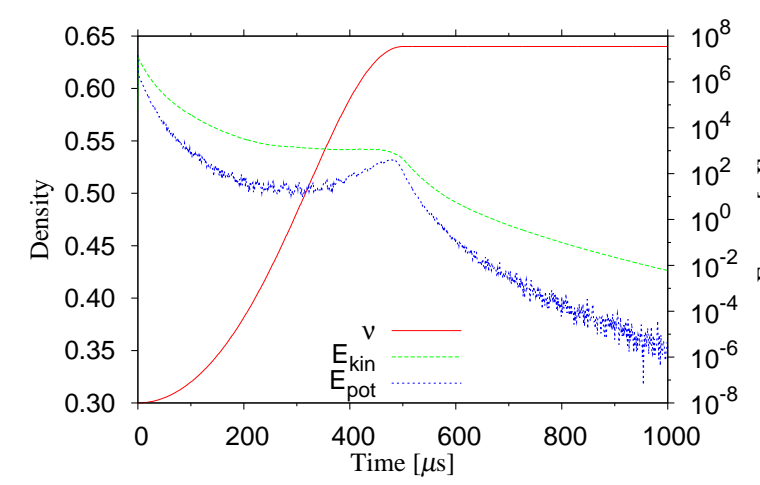

(e)

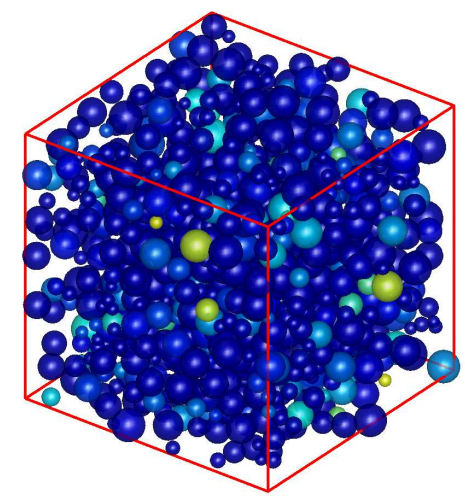

(b)

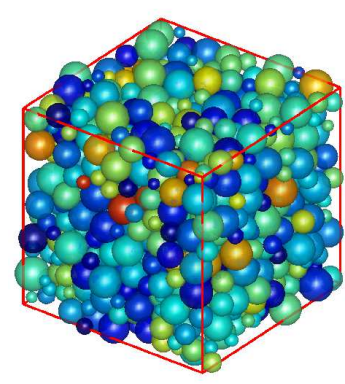

(d)

Figure 2. Snapshots of the (a) initial (fully) random configuration of the particles with big (artificial) overlaps and (b) the situation after only $40 \mu$ seconds compression when all artificial overlaps have disappeared. The color code indicates overlaps of the particles (red: big overlaps, blue: no overlap). (c) Snapshot of the relaxed granular "fluid" with volume fraction $\nu_{\mathrm{i}}=0.64$. Note that although particles are densely packed they have still practically no overlap, since the volume fraction is below the jamming value $\nu_{\mathrm{j}}$. (d) Snapshot of the strongly compressed packing, with $\nu_{\max }=0.75$ using the same color code as in (a), (b) and (c). (e) Evolution of the volume fraction, the potential and the kinetic energy during initial compression and relaxation and (f) the loading-unloading cycle. 
all of their contacts.

Although it is possible to check numerically the contribution of every particle to the force network [25] an easier although less rigorous way to identify rattlers is to just count their contacts. Since frictionless particles with less than four contacts are not mechanically stable they are defined as rattlers. This leads to the following abbreviations and definitions as used in the rest of this study.

$$
\begin{aligned}
N & : \text { Total number of particles. } \\
N_{4}:=N_{C \geq 4} & : \text { Number of particles with at least } 4 \text { contacts. } \\
M & : \text { Total number of contacts } \\
M_{4}:=M_{C \geq 4} & : \text { Total number of contacts of particles with at least } 4 \text { contacts. } \\
C^{r}:=\frac{M}{N} & : \text { Coordination number (classical definition). } \\
C:=C^{m}=\frac{M_{4}}{N} \quad: & \text { Coordination number (modified definition). } \\
C^{*}:=\frac{M_{4}}{N_{4}}=\frac{C}{1-\phi_{r}}: & \text { Corrected coordination number. } \\
\phi_{r}:=\frac{N-N_{4}}{N}: & \text { (Number) fraction of rattlers. } \\
\nu:=\frac{1}{V} \sum_{p \in N} V_{p}: & \text { Volume fraction of the particles. } \\
\nu^{*}:=\nu-\nu_{r}=\frac{1}{V} \sum_{p \in N_{4}} V_{p} & : \text { Volume fraction of the particles excluding rattlers. } \\
\nu_{r}:=\frac{1}{V} \sum_{p \notin N_{4}} V_{p}: & \text { Volume fraction of rattlers. }
\end{aligned}
$$

The difference between the coordination numbers $C^{r}$ and $C$ is not caused by the "ideal rattlers" with $C=0$, since those do not contribute to $C$ anyway. It is caused by those particles (virtual, dynamic rattlers) with $1 \leq C \leq 3$, which are not mechanically stable, i.e., temporary, members of the contact network. They are neglected when counting the contacts $M_{4}$. In the following, we will use the modified coordination number $C:=C^{m}$, instead of $C^{r}$, since it better resembles the slow, quasi-static deformation mode of the system, as will be discussed below.

The ratio of $M_{4}$ and $N_{4}$ provides the corrected coordination number $C^{*}$, which perfectly follows the isostaticity arguments. The fraction of rattlers and a comparison between the classical, the modified and the corrected definitions are shown in Fig. 3. The values of $C^{r}$ and $C^{m}$ are very similar, since the number of contacts originating from particles with $C=1,2$, or 3 contacts is small anyway and decays with decaying rate of deformation. As to be expected, the value of $C^{*}$ is considerably larger and all coordination numbers display a sharp jump at the jamming transition during un-loading. In the left panel, Fig. 3(a), the respective fractions of particles with different numbers of contacts are shown, where the red solid line represents $\phi_{r}$. Coming from high densities, the fraction of rattlers increases and jumps to unity when approaching $\nu_{r}$. In the right panel, Fig. 3(b), the different versions of the coordination numbers are compared, showing that, while the loading and unloading branch are clearly different, $C^{r}$, and $C$, are only slightly different close to and below the critical volume fraction $\nu_{c}$. Even though larger, $C^{*}$ behaves qualitatively similar below and above the jamming transition.

However, since $C^{*}$ involves not all particles, it cannot easily be related to the total particle volume, or the mass-density of the system - that is equivalent to the volume fraction, i.e., $\rho=\rho^{p} \nu$, with the particle 


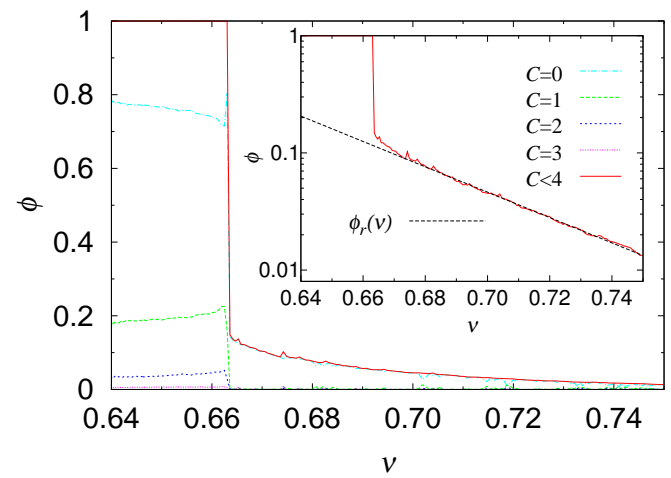

(a)

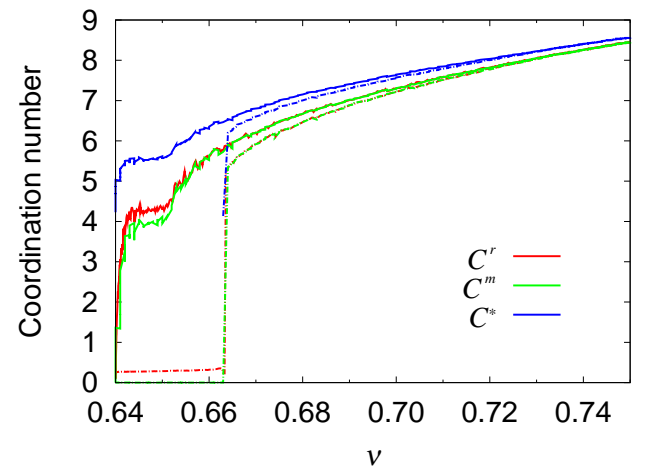

(b)

Figure 3. (a) Evolution of the fraction of rattlers as function of volume during fraction during unloading for a simulation with $N=10000, w=3$, and $D=0.001$. Inset: Fit of Eq. (3). (b) Comparison of the coordination numbers computed using the classical $C^{r}$, the modified $C$ and the corrected $C^{*}$, for the same simulation. The data for loading and unloading are shown by solid and dashed lines, respectively.

material density $\rho^{p}$-as experimentally accessible for many systems. The average contact number density $\nu C$ can be related to the mechanically relevant contact number density $\nu^{*} C^{*}$ (without rattlers):

$$
\nu C=\frac{N\left\langle V_{p}\right\rangle}{V} C=\frac{\left(1-\phi_{r}\right) N\left\langle V_{p}\right\rangle}{V} \frac{C}{1-\phi_{r}}=\left(1-\phi_{r}\right) \nu C^{*} \neq \nu^{*} C^{*}=\left(\nu-\nu_{r}\right) C^{*},
$$

where $V$ is the volume occupied by the packing. The non-equality could become an equal only if the average volume of rattlers is equal to the average volume of all particles, i.e., if $\nu_{r} / \nu=\phi_{r}$. Unfortunately, there is no simple exact relation between $\nu C$ and $\nu^{*} C^{*}$, as discussed below in section 4 , since the smaller particles are more likely to be rattlers. Therefore, we will work with the parameters $\nu, C^{*}(\nu)$ (see below), and $\phi_{r}(\nu)$.

The fraction of rattlers, in the quasi-static limit, i.e., for extremely slow deformations, as presented below, obeys the empirical relation:

$$
\phi_{r}(\nu)=\phi_{c} \exp \left[-\phi_{\nu}\left(\frac{\nu}{\nu_{c}}-1\right)\right]
$$

for $\nu \geq \nu_{c}$ and $\phi_{r}\left(\nu<\nu_{c}\right)=1$ otherwise. This involves two fit parameters (i) the fraction of rattlers at jamming, $\phi_{c}$, and (ii) the rate of decay of rattlers with increasing packing fraction, $\phi_{\nu}$. A fit of $\phi_{r}(\nu)$ is shown in the inset of Fig. 3(a). Note that $\nu_{c}$ cannot be obtained by the fit like Eq. (3), but has to be obtained by other means [14], e.g., by identifaction of the jump/discontinuity of $\phi_{r}\left(\nu_{c}\right)$. Typical values are $\phi_{c} \approx 0.13 \pm 0.03$ and $\phi_{\nu} \approx 15 \pm 2$. The observation that one has $\phi_{r}\left(\nu_{\mathrm{RLP}}\right) \approx 1$ at the random loose packing fraction $\nu_{\mathrm{RLP}} \approx 0.57$ is presumably accidental.

The corrected coordination number $C^{*}$, obtained by disregarding rattlers, obeys a power law of volume fraction as reported previously $[6,7,15,24]$ :

$$
C^{*}(\nu)=C_{0}+C_{1}\left(\frac{\nu}{\nu_{c}}-1\right)^{\alpha},
$$

where $\nu_{c}$ is the critical volume fraction, $C_{0}$ is the critical coordination number, and $C_{1}$ is the prefactor for the power-law with power $\alpha$. Given $C_{0}=4,6$ in two and three dimensions, for isostatic packings of frictionless particles, this would leave three more fit parameters (iii) $\nu_{c} \approx \nu_{\mathrm{RCP}}$, (iv) $C_{1} \approx 8$, and (v) 
$\alpha \approx 0.5$. However, we sometimes allow also $C_{0}$ as free parameter in order to check the consistency with the isostaticity assumption for the packings.

Below we check this analytical expression for $C^{*}(\nu)$ for the un-loading branch of our simulations, since these data show much less dynamical artefacts than data from the loading branch. We do not discuss cyclic loading and un-loading, which can lead to a continuous "drift" (increase) of $\nu_{c}$ with each loading cycle [26]. Within the present paper, the hysteresis under cyclic loading, and possible quantitative information that can be extracted from it (as, e.g., in magnetic systems), is not studied in detail.

Note that we do not identify the $\nu_{c}$ for un-loading with the jamming volume fraction $\nu_{j}$. Actually, we doubt that there is one jamming volume fraction. The critical value rather depends on the contact properties and on the history of the packing, especially when realistic properties like friction are involved, but also for the frictionless case studied here. A detailed study of the dependence of $\nu_{c}$ on the contact properties and on the history of the packing in general is far from the scope of this study, so that we focus mainly on the first un-loading branch.

\subsection{Influence of polydispersity}

In order to understand the effect of polydispersity, we first perform simulations using three rather small packings of 1000 particles with three different widths of the size distribution $w=1,2,3$. These samples are compressed and then decompressed, at the same rate, between $\nu_{\mathrm{i}}=0.5$ and $\nu_{\max }=0.9$. Figure 4 displays the relation between volume fraction and coordination number for these packings. The finite values of the coordination number during compression, at low densities, make the transition from fluid to solid state difficult to detect. This is due to temporary contacts which arise from the dynamics at low densities. If the packing is allowed to relax the dynamic contacts become less and the state of zero coordination is approached, as expected. ${ }^{1}$ However, not even our slowest simulations allowed us to avoid dynamic contacts in the compression branch.

On the other hand, a much cleaner, very sharp decrease in $C$ is observed during un-loading (decompression), when we approach $\nu_{c}$ from high densities, see Fig. 4. The fit of Eq. (4) to the corrected coordination number, $C^{*}$, computed during decompression, is shown in the inset of Fig. 4. The transition from the jammed to the unjammed state occurs at higher volume fractions for more polydisperse, heterogeneous packings. A list of the numerical values of the fit parameters is given in table 1 .

Even though the system is rather small and the deformation rate is rather high, the fitted parameters are almost consistent with the isostaticity assumption, $C_{0}=6$. When this is imposed, the fit parameters are quite close to each other and become almost independent of $w$. Only for $\nu_{c}$ there is an increasing trend for increasing $w$.

1. Remark on the fit of Equation (4). We choose to fit Eq. (4) to the decompression branch of the simulation data because the system's kinetic to potential energy ratio is much lower than during compression in this density range, see Fig. 2(f), even for the rather fast compression used. Furthermore, boundary effects are less important during decompression because the system is expanding and possible spurious contacts caused by the (virtual, periodic) wall motion are avoided. In a separate set of simulations, we find that by adding extra relaxation between deformation steps, the compression and decompression branches of $C(\nu)$ can get closer to each other (data not shown). The distance between the branches reduces with the relaxation step but does not disappear even for the largest relaxation-times. Since the unloading branch is much less sensible to the protocol and rate of deformation, from now on, we will fit Eq. (4), i.e., the analytical expression of the corrected coordination number, exclusively to the decompression branch of the simulation data. 


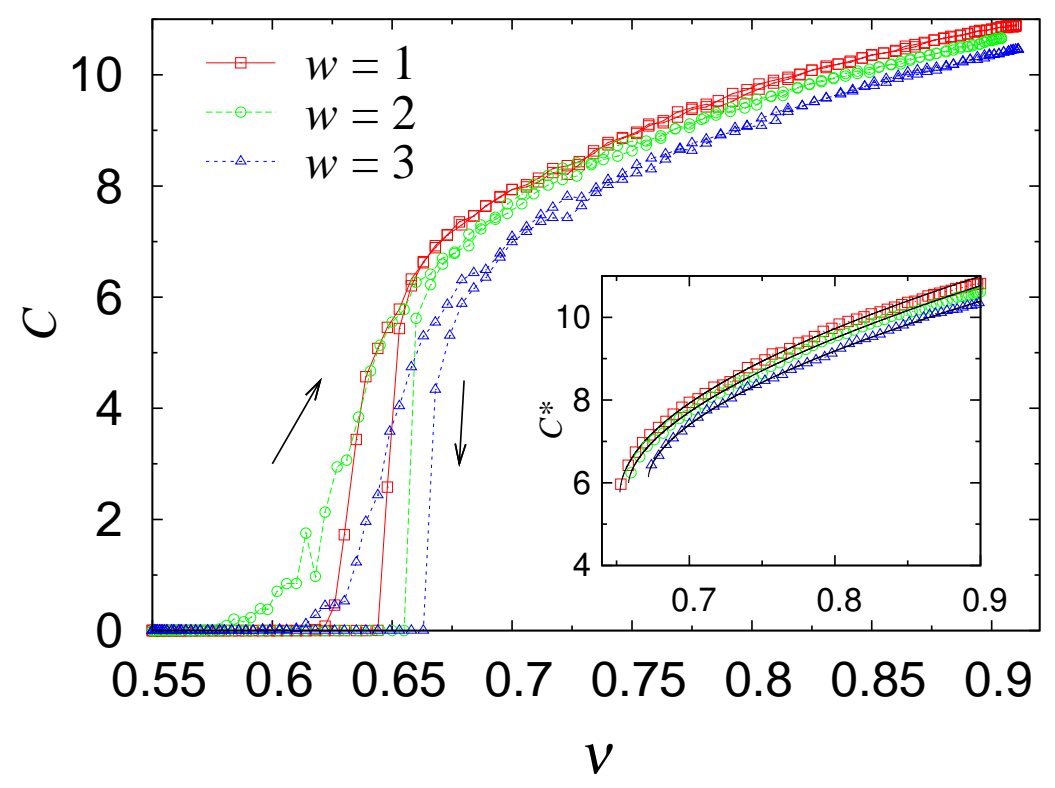

Figure 4. Coordination number $C$ as function of volume fraction $\nu$ for packings of 1000 particles with different size distributions of width $w$, as given in the figure. The arrows indicate the compression (up) and decompression (down) directions. Inset: The lines are fits of the corrected coordination number according to Eq. (4), with the fit-parameters given in table 1.

\subsection{History and system size dependence}

It is especially interesting to see how simulation parameters such as deformation history and system size affect jamming and the evolution of the coordination number. We studied the effect of deformation history by compressing and decompressing isotropically two packings with 1000 particles and polydispersity $w=$ 3 , but for different volume fraction ranges. The first sample is compressed from an initial state close to jamming up to a very high volume fraction $(\nu: 0.64 \rightleftarrows 0.9)$ and back. The second sample is compressed from the same initial state up to a moderate volume fraction $(\nu: 0.64 \rightleftarrows 0.75)$ and back.

Figure 5(a) shows the evolution of the coordination number as function of $\nu$ for both samples. Although, the highly compressed packing seems to have a larger critical volume fraction, the difference practically disappears when rattlers are removed. Figure 5(b) shows the corrected coordination number $C^{*}$ during decompression and the fit of Eq. (4) to the data obtained from the moderately compressed sample. Note that the fit is also quite good as an extrapolation for stronger compression, i.e., higher densities, suggesting that isotropic deformation history has no substantial effect on the coordination number at higher volume fractions.

The size of the system has no effect on the critical volume fraction and the evolution of the coordination number. Figure 6 illustrates the coordination number as function of volume fraction during a cycle of compression-decompression for three packings comprising $N=1000,5000$ and 10000 particles. All samples are deformed at the same relative rate $D=0.5$, with the same polydispersity parameter $w=3$. The small size systems show stronger fluctuations prior to jamming since dynamical effects are more pronounced for. On the other hand, after jamming all curves obey a similar power law as confirmed by the fits of Eq. (4) to the corrected coordination number $C^{*}$, shown in the inset of Figure 6.

The values of the critical volume fractions obtained from the fits are $0.6650 \pm 0.0002,0.6647 \pm 0.0001$, 


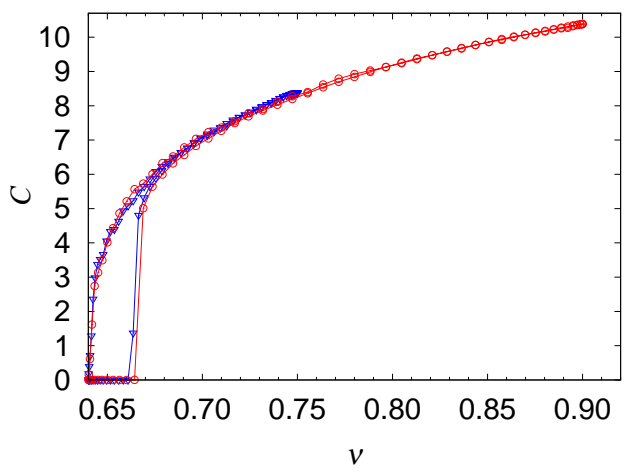

(a)

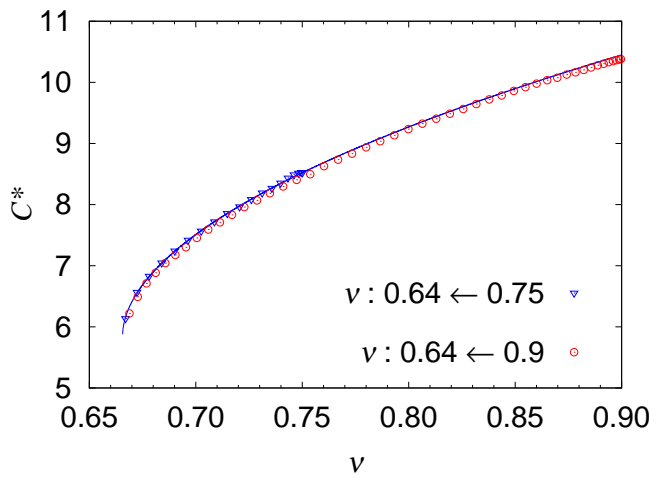

(b)

Figure 5. (a) Coordination number $C$ as function of volume fraction $\nu$ for different compression histories. (b) Evolution of $C^{*}$ during decompression. The solid line is the fit of Eq. (4) to the data obtained from the moderately compressed sample $(\nu: 0.64 \rightleftarrows 0.75)$.

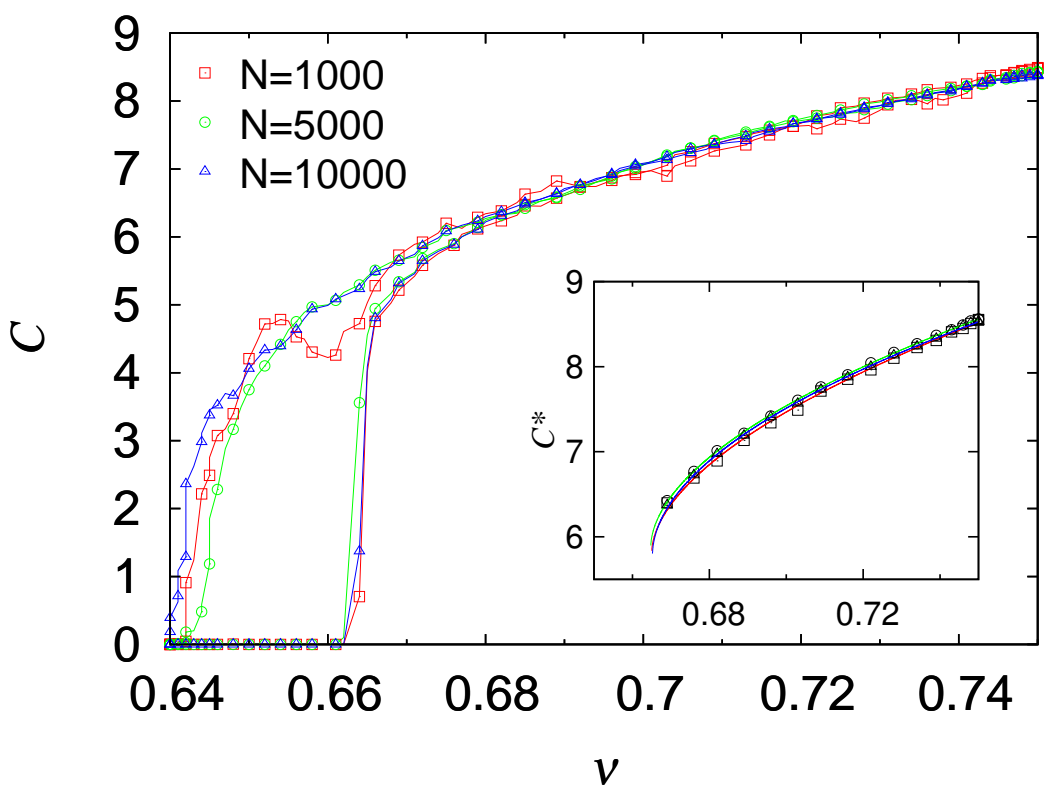

Figure 6. Evolution of the coordination number for different system sizes, with $w=3$ and $D=0.5$. Inset: Fits of the corrected coordination number $C^{*}$ according to Eq. (4). The red, green and blue lines are the fits for $N=1000,5000$ and 10000, respectively.

and $0.6652 \pm 0.0001$, for $N=1000,5000$, and 10000, respectively. The other parameters, see Table 2 , are very close to each other and to those reported in Table 1 . These rather small differences between the critical volume fractions (and also the other fit parameters) for different $N$ imply that the system size does not have an important effect on the evolution of the (corrected) coordination number $C^{*}$. Larger systems display smaller statistical fluctuations, however. 


\subsection{Effect of loading rate}

The effect of the loading rate on jamming and the evolution of the coordination number is analyzed by applying isotropic deformation to a polydisperse $(w=3)$ sample at various rates. Figure 7 (a) shows the evolution of the coordination number as function of volume fraction for a packing of 10000 particles deformed at relative rates $D=1,0.5,0.1,0.01$, and 0.001 . The fits of Eq. (4) to the corrected coordination number are shown in Fig. 7(b) and the fit parameters are summarized in table 2.

The jamming transition should best be studied in the quasi-static limit, i.e., for $D \rightarrow 0$, when the sample has infinitely long time to relax. However, practically, this is impossible [15]. Using the fit of Eq. (4) for a systematic study of the deformation rate effect on the critical volume fraction is not reliable due to the singularity of its derivative at this point. The rapid change of the slope of $C^{*}(\nu)$ near jamming increases the sensitivity of other parameters to the fit range and causes them to fluctuate. When studying the jamming transition, in recent studies, the densities very close to $\nu_{c}$ were carefully studied. Note that here, we provide data for a much wider range of densities, far away from the transition - to be used for practical applications. Therefore, the parameters and especially the exponents reported in this study can be slightly different from those in previous studies.

For example, the exponent $\alpha \simeq 0.5$ previously reported in [6] for $2 \mathrm{D}$ and $[7,15]$ for $3 \mathrm{D}$, cannot be always recovered (see Table 2) for very slow compression; we rather find $\alpha \simeq 0.66$ for the slowest compression rates. The critical volume fraction, on the other hand, is not varying much and these variations are presumably due to the sensitive fit function with a singular slope close to $\nu_{c}$, as mentioned already above. In Ref. [14], alternative methods were compared to determine the critical volume fraction based on the fraction of rattlers, the pressure, and the ratio of the kinetic and potential energies of the packing. For a better, more objective analysis of rate effects, we believe that the fit should be used in conjunction with at least one of these methods. Then, when obtained independently, $\nu_{c}$ is not a free fit parameter anymore. However, since changing the loading rate seems to have no strong effect on $\nu_{c}$, and the coordination numbers at volume fractions considerably above $\nu_{c}$, we do not pursue this further.

\section{Fabric Tensor}

In the following, we compare the simulation results on the trace of the fabric tensor to the recent 3D predictions of Durán et al. [22] that complement the older 2D results by Madadi et al. [17, 19]. In these studies, the effect of polydispersity on the trace of the fabric tensor was expressed in terms of the moments of the size distribution. The basic assumption, in both $2 \mathrm{D}$ and $3 \mathrm{D}$, is that the linear compacity $c_{s}$, defined as the fraction of the particle surface shielded by its neighbors, is independent of the particle radius. From this the trace of the fabric is found to be proportional to the contact number density, $\nu C$, and a dimensionless pre-factor (see $g_{3}$ below) that only depends on the moments of the size-distribution. Since derivation is similar in both $2 \mathrm{D}$ and $3 \mathrm{D}$, only some formulas are shown; for more details we refer to Refs. $[17,19,22]$.

As first order approximation, in $3 \mathrm{D}$, the mean number of contacts, $C(r)$, of a particle with radius $r$ is inversely proportional to the fraction of its surface $\Omega(r) /(4 \pi)$ shielded by a neighboring sphere of characteristic radius $\langle r\rangle$, such that:

$$
C(r)=\frac{4 \pi c_{s}}{\Omega(r)},
$$

where $\Omega(r)=2 \pi(1-\cos \alpha)$, with the sinus and cosinus of the shielding half-angle, $\sin \alpha=1 /(r /\langle r\rangle+$ 1) and $\cos \alpha=\sqrt{1-\sin ^{2} \alpha}$, respectively. When inserting Eq. (5) into the definition of the average 


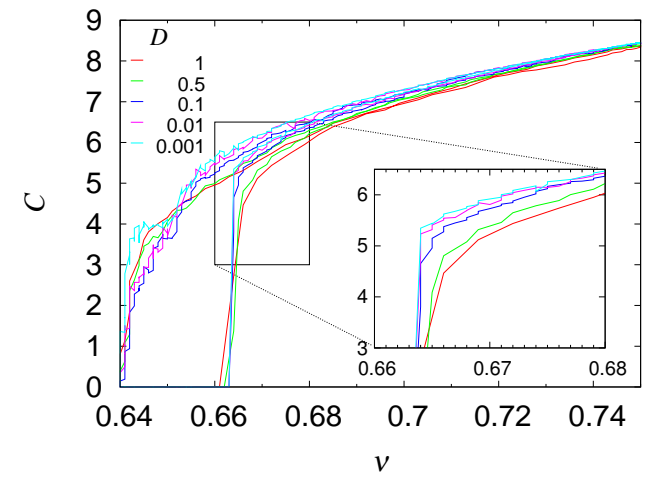

(a)

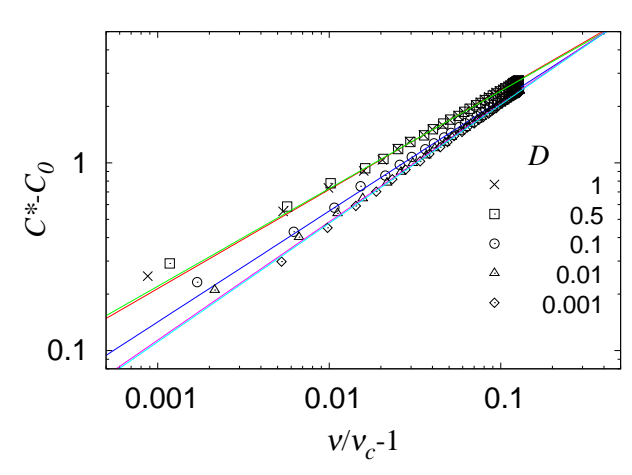

(c)

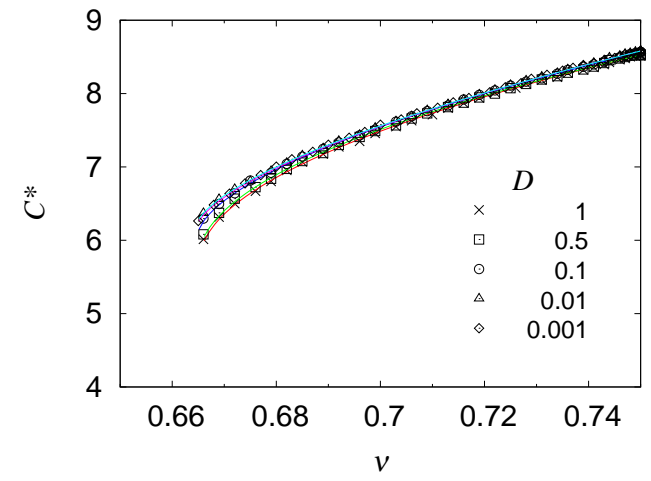

(b)

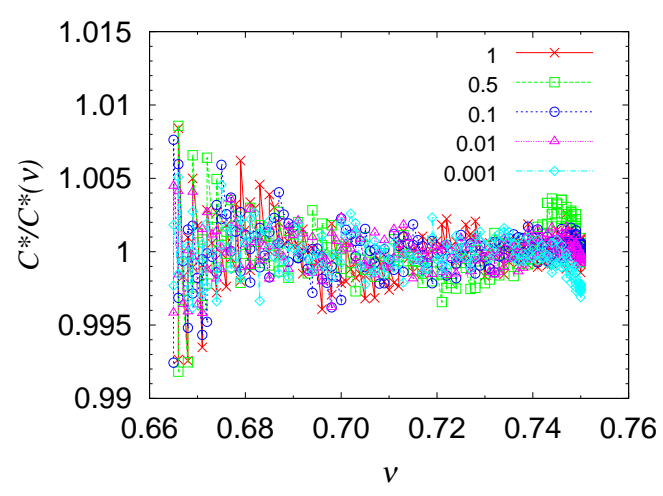

(d)

Figure 7. (a) Evolution of the coordination number for different deformation rates. Inset: Zoom into the decompression branch during transition from the jammed to the unjammed state. (b) The corrected coordination number $C^{*}$ and the fits of Eq. (4). (c) Log-log plot of $C^{*}-C_{0}$ against $\left(\nu / \nu_{c}-1\right)$ from the same data as in (a) and (b). (d) The ratio of data and fit, $C^{*} / C^{*}(\nu)$, indicates that the quality of the fit is better than one percent for the full range of data $\left[\nu_{c} ; 0.75\right]$.

coordination number $C=\int_{0}^{\infty} C(r) f(r) d r=4 \pi c_{s} \int_{0}^{\infty}[f(r) / \Omega(r)] d r$, it is possible to calculate explicitly the expected compacity for different $C$ :

$$
c_{s}(C)=\frac{a_{2} C}{1-C_{2}+C_{2} \hat{r}_{2}}
$$

with the dimensionless second moment $\hat{r}_{2}$ from Eq. (2). Using the quadratic approximation of Durán et al. [22] for the solid angle $\Omega(r)$ leads to $a_{2}=\Omega(\langle r\rangle) /(4 \pi)=\frac{1}{2}(1-\sqrt{3} / 2), B_{2}=\sqrt{3} / 24 a_{2}$, and $C_{2}=B_{2}\left(B_{2}-5 / 6\right)$. For example, in the monodisperse special case one has $c_{s}=a_{2} C$, so that inserting the isostatic limit $C^{*}=C\left(1-\phi_{c}\right)=6$ leads to $c_{s}=6 a_{2} /\left(1-\phi_{c}\right) \approx 0.47$ for $\phi_{c} \approx 0.15$, i.e., about half of the surface of particles is shielded close to the jamming point.

Figure 8 shows the numerical data for the coordination number $C(r)$ and the compacity $c_{s}(r)$ as function of $r /\langle r\rangle$ for $w=3$ (for which $\hat{r}_{2}=13 / 12$ ) and two different volume fractions: a very high one $(\nu \approx 0.74)$ and one close to jamming $(\nu \approx 0.67)$, along with the predicted relations from Eqs. $(5)$ and (6), for coordination number and compacity, respectively. Although, Eq. (5) describes the size-dependent contact number qualitatively well for a broad range of densities, at small radii, the contact number drops 
considerably below the predictions, see Figs. 8(a) and 8(c). The assumption of a constant compacity is confirmed for the larger particle radii, but fails for smaller radii, see Figs. 8(b) and 8(d).

Using the average coordination number, $C$, or inserting $C^{*}=C /\left(1-\phi_{r}\right)$ into Eq. (6) leads to the red and blue data sets, respectively. Clearly the theoretical prediction that uses $C$ is superior to the one using $C^{*}$. Nevertheless, we report the interesting and intuitive observation that the latter coordination number has a lower limit $C^{*}(r) \geq 4$, since rattlers are excluded. Since small particles have smaller surface area, their chance to have less than four contacts is higher, so that more rattlers are from the small fractions. Interestingly, the data for $c_{s}(r)$ indicate that those small particles that are not rattlers have a higher compacity than the average. Different shapes and wider size distributions have to be studied to allow more general insights.

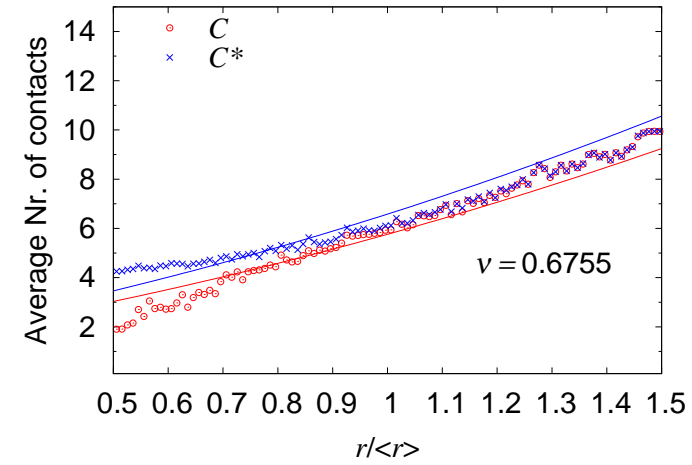

(a)

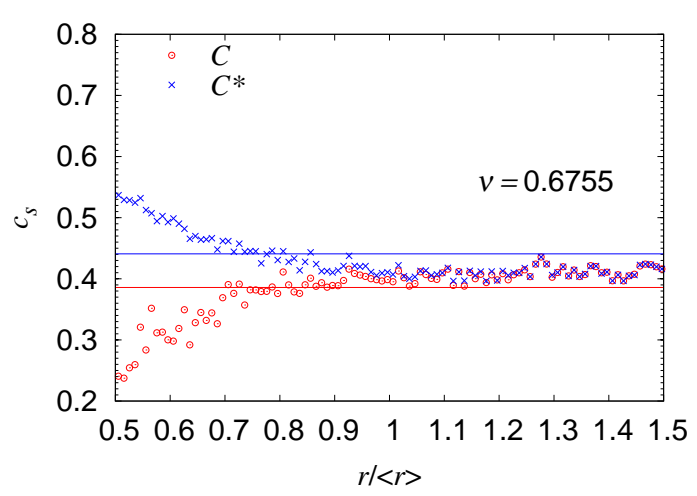

(c)

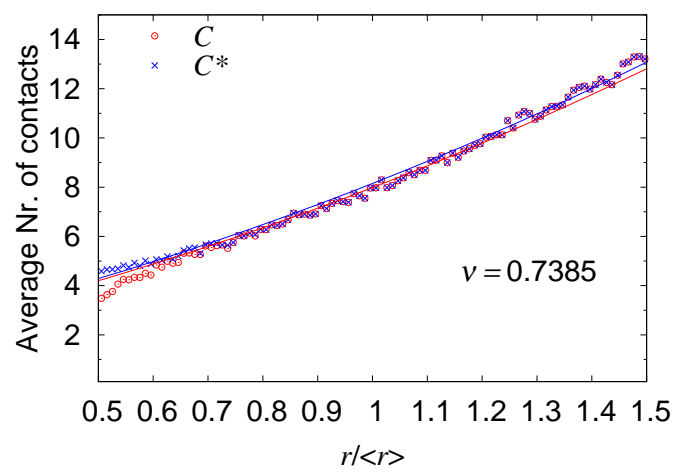

(b)

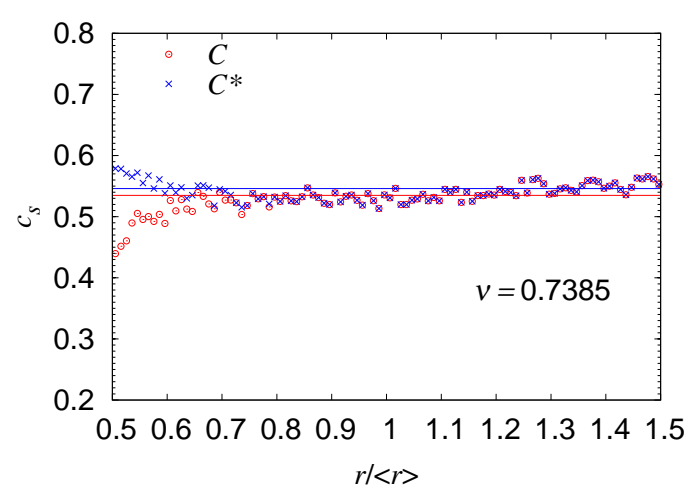

(d)

Figure 8. (a,b) Average number of contacts $C(r)$ as function of the normalized particle radius, including (red) and excluding (blue) rattlers, at different volume fractions for packings with $N=10000$ particles. The points are data from the simulations while the solid lines are the analytical predictions of Eq. (5) using either $c_{S}(C)$ (red) or $c_{s}\left(C^{*}\right)$ (blue), and thus confirming that using $\left.c_{s}(C)=c_{s}\left(\left(1-\phi_{r}\right) C^{*}\right)\right)$ in Eq. (6) is self-consistent. (c,d) Linear compacity $c_{s}$ as function of the normalized radius, computed from the same packings as in (a) and (b), including (red) and excluding (blue) rattlers. Again the solid lines are the theoretical prediction of Eq. (6).

Using the definition of the average coordination number, $C$, the trace of the fabric can be written as detailed in Ref. [22]: 


$$
F_{V}=\operatorname{tr}(\mathbf{F})=(1 / V) \sum_{p \in V} V_{p} C_{p}=(N / V) \int_{0}^{\infty} d r V_{p}(r) C(r) f(r)=g_{3} \nu C,
$$

with the volumes $V_{p}$ and the contact numbers $C_{p}$ of particles $p$, and the term $g_{3}$, which contains the information about the polydispersity, which is defined as [22]:

$$
g_{3}=\frac{\left\langle r^{3}\right\rangle_{\Omega}}{\left\langle r^{3}\right\rangle}=\frac{\int_{0}^{\infty} r^{3}[f(r) / \Omega(r)] d r}{\left\langle r^{3}\right\rangle \int_{0}^{\infty}[f(r) / \Omega(r)] d r},
$$

where the brackets $\langle\ldots\rangle_{\Omega}$ indicate the normalized averaging over the modified distribution function $[f(r) / \Omega(r)]$. Using the moment expansion of Durán et al. [22], the lowest order analytical approximation (that involves moments up to order $k=5$ ) is:

$$
g_{3} \approx \frac{1-B_{2}+C_{2}+\left(B_{2}-2 C_{2}\right) \frac{\left\langle r^{4}\right\rangle}{\langle r\rangle\left\langle r^{3}\right\rangle}+C_{2} \frac{\left\langle r^{5}\right\rangle}{\langle r\rangle^{2}\left\langle r^{3}\right\rangle}}{1+C_{2}\left[\frac{\left\langle r^{2}\right\rangle}{\langle r\rangle^{2}}-1\right]}
$$

where the constants $B_{2}$ and $C_{2}$ were defined in the previous section. This is considerably more involved than the $2 \mathrm{D}$ results $[17,19]$, since none of the above terms can be neglected [22]. Only for the monodisperse situation, one has the simplification $g_{3}=1$.

Equation (7) is plotted in Fig. 9 using the simulation data for different distribution widths $w$. For all distributions and packing densities from very loose up to very dense packings $(\nu \sim 0.9)$, the proportionality between the trace of the fabric and the contact density is well described by Eq. (9), when the correction factor $g_{3}$ is used. More explicitly, the correction factor, even though not perfect, improves the quality of the prediction considerably. The reason for the remaining disagreement of order of $1 \%$ can be due to the assumption of particles of radius $r$ being surrounded by particles of mean radius, due to neglecting the overlap of the particles in the theoretical considerations, or due to the higher proability for small particles to be rattlers.

The moments of the size distribution can be expressed in terms of the relative width $w$ using Eq. (2), which allows us to study the behavior of $g_{3}$ as a function of $w$. The inset of Fig. 9 shows the analytical approximation and the exact definition of $g_{3}$, from Eq. (8), along with the values of $g_{3}$ obtained from the DEM simulation. For highly polydisperse packings, corresponding to large $w$, the $k$ th moment becomes $\left\langle r^{k}\right\rangle \rightarrow\langle r\rangle^{k} 2^{k} /(k+1)$ and $g_{3}$ thus saturates at a constant $g_{3}^{\max } \approx 1.62$. Therefore, the influence of an increase in the polydispersity on $\operatorname{tr}(\mathbf{F})$ is limited for high $w$ in the framework of the approximations made. A more detailed study of this prediction for wide size distributions is, however, far from the scope of this study.

\section{Pressure}

In this section, the pressure is introduced and related to the other system properties volume fraction, coordination number, fraction of rattlers, and fabric. In order to better understand the final analytical expressions, the stress is rewritten and re-phrased, starting from the traditional definitions.

The micromechanical stress tensor components for a (static) particle (in mechanical equilibrium) are defined as:

$$
\sigma_{i j}^{p}=\frac{1}{V_{p}} \sum_{c=1}^{C_{p}} l_{i}^{p c} f_{j}^{p c}
$$




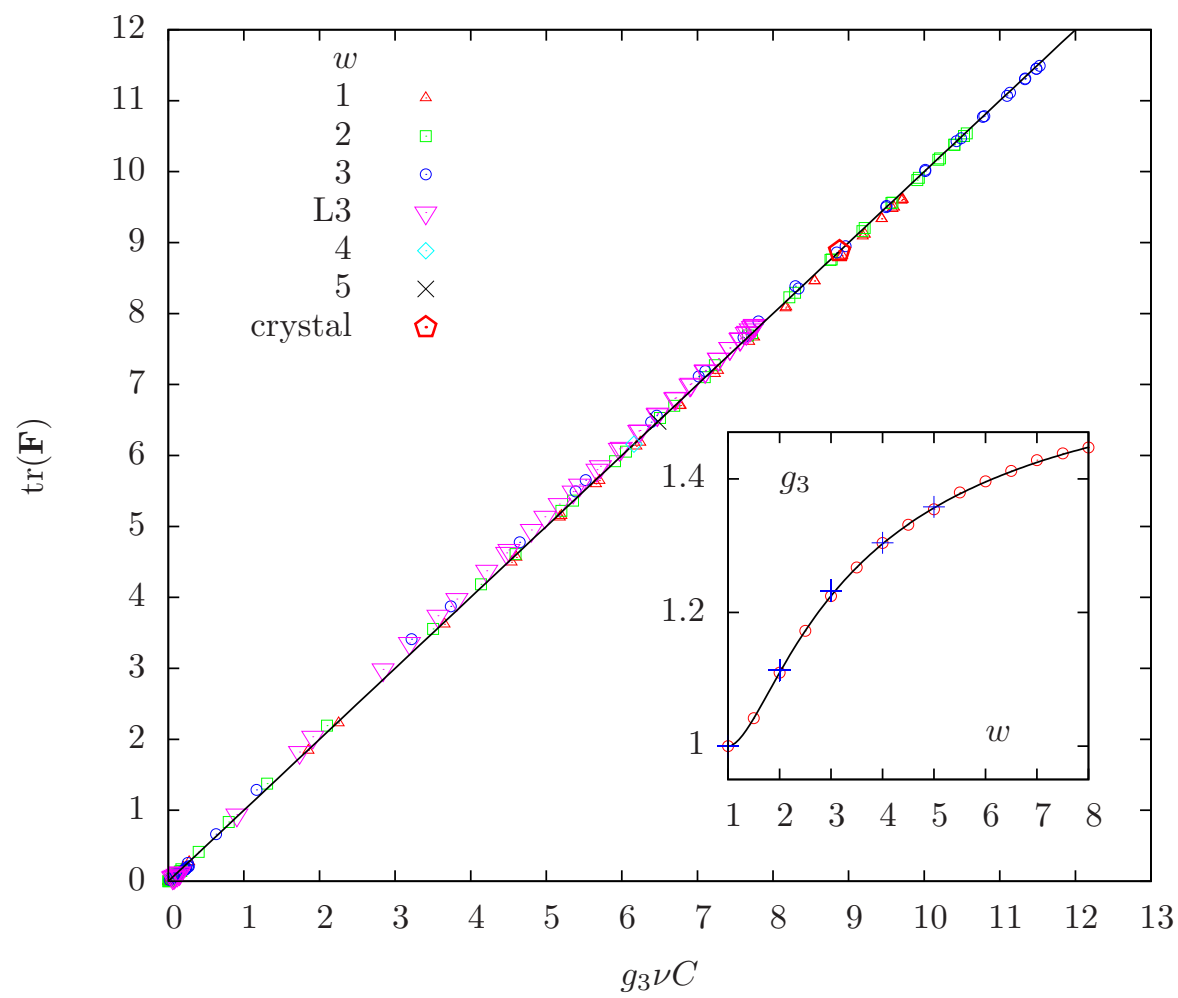

Figure 9. The trace of the fabric tensor as given by Eq. (7) for different size distributions with $w$ given in the inset from simulations with $N=1000$ ("L3" indicates a larger simulation with $N=10000$ and "crystal" indicates an ordered lattice structure whereas $w=1$ is a disordered, monodisperse configuration). Each data-point corresponds to one density and fabric, as averaged over the whole system, at different densities during decompression. Inset: The constant $g_{3}$ plotted as function of $w$ from its definition (o), the analytical approximation (solid line) and the simulation data $(+)$.

where $\mathbf{l}^{p c}=\left(r_{p}-\delta_{c} / 2\right) \hat{\mathbf{n}}$ is the branch vector of contact $c$ and $\mathbf{f}^{p c}=k_{n} \delta_{c} \hat{\mathbf{n}}$ is the (linear) force associated, with particle radius, $r_{p}$, overlap $\delta_{c}$, spring-stiffness, $k_{n}$, and the contact-direction unit vector, $\hat{\mathbf{n}}$. Here we assume [4] that the contact point is located at the middle of the overlap. ${ }^{2}$ From these definitions, the trace of the stress for a single particle becomes:

$$
\operatorname{tr}\left(\boldsymbol{\sigma}^{p}\right)=\frac{k_{n}}{V_{p}} \sum_{c=1}^{C_{p}} \delta_{c}\left(r_{p}-\frac{\delta_{c}}{2}\right),
$$

with the number of contacts $C_{p}$ of particle $p$. For a packing of $N$ particles, the trace of the average stress tensor can be computed by weighing the particles according to their volume [27]:

2. A more realistic alternative would be to define it on the plane bisecting the particles in contact and split the overlap accordingly, however, the accuracy gained in doing so would be negligible for small overlaps and similar particle radii. 


$$
\begin{aligned}
\operatorname{tr}(\boldsymbol{\sigma}) & =\frac{1}{V} \sum_{p \in V} V_{p} \operatorname{tr}\left(\boldsymbol{\sigma}^{p}\right) \\
& =\frac{k_{n}}{V} \sum_{p=1}^{N}\left(r_{p} \sum_{c=1}^{C_{p}} \delta_{c}-\frac{1}{2} \sum_{c=1}^{C_{p}} \delta_{c}^{2}\right),
\end{aligned}
$$

where $V$ is the total volume of the packing.

One can express $V$ in terms of the volume fraction and the volume of the $N$ particles as $V=N\left\langle V_{p}\right\rangle / \nu$, with $\left\langle V_{p}\right\rangle=\frac{4 \pi}{3}\left\langle r_{p}^{3}\right\rangle$, where the brackets denote averaging of a particle-property $A_{p}$ over all particles in a packing, e.g., $\langle A\rangle:=\left\langle A_{p}\right\rangle=\frac{1}{N} \sum_{p=1}^{N} A_{p}$. Introducing also the normalized average normal force for each particle $p$ as $\phi_{p} \equiv f_{p} /\left\langle f_{p}\right\rangle$, with $f_{p}=\sum_{c=1}^{C_{p}} k_{n} \delta_{c}$, the trace of the averaged stress tensor becomes:

$$
\begin{aligned}
\operatorname{tr}(\boldsymbol{\sigma}) & =\frac{3 k_{n} \nu}{4 \pi\left\langle r^{3}\right\rangle} \frac{1}{N} \sum_{p=1}^{N}\left(r_{p} \sum_{c=1}^{C_{p}} \delta_{c}-\frac{1}{2} \sum_{c=1}^{C_{p}} \delta_{c}^{2}\right) \\
& =\frac{3 k_{n}}{4 \pi} \frac{\nu}{\left\langle r^{3}\right\rangle}\left(\left\langle\sum_{c=1}^{C_{p}} \delta_{c}\right\rangle\left\langle r_{p} \phi_{p}\right\rangle-\frac{1}{2}\left\langle\sum_{c=1}^{C_{p}} \delta_{c}^{2}\right\rangle\right) \\
& =\frac{3 k_{n}}{4 \pi} \frac{\nu C\langle\delta\rangle_{c}}{\left\langle r^{3}\right\rangle}\left(\left\langle r_{p} \phi_{p}\right\rangle-\frac{\left\langle\delta^{2}\right\rangle_{c}}{2\langle\delta\rangle_{c}}\right)
\end{aligned}
$$

where $C=\frac{M_{4}}{N}=\frac{1}{N} \sum_{p \in N_{4}} C_{p}$ is the mean coordination number (or just coordination number, averaged over all particles), $\langle\delta\rangle_{c} \equiv \frac{1}{M_{4}} \sum_{c \in M_{4}} \delta_{c}$ is the average overlap over all $M_{4}$ contacts, of particles with four or more contacts that contribute to the contact network, and we have used the identities: $\left\langle\sum_{c=1}^{C_{p}} \delta_{c}\right\rangle \equiv C\langle\delta\rangle_{c}$ and $\left\langle\sum_{c=1}^{C_{p}} \delta_{c}^{2}\right\rangle \equiv C\left\langle\delta^{2}\right\rangle_{c}$.

The non-dimensional pressure is defined as $p=\frac{2\langle r\rangle}{3 k_{n}} \operatorname{tr}(\sigma)$, so that introducing the normalized particle radius $\xi_{p}=r_{p} /\langle r\rangle$ and overlap $\Delta_{c}=\delta_{c} /\langle r\rangle$ leads to:

$$
p=p\left(\langle\Delta\rangle_{c}\right)=\frac{1}{4 \pi} \nu C\langle\Delta\rangle_{c}\left(2 g_{p}-b\langle\Delta\rangle_{c}\right)
$$

where the factors are

$$
g_{p}=\frac{\left\langle\xi_{p} \phi_{p}\right\rangle}{\left\langle\xi^{3}\right\rangle} \quad \text { and } \quad b=\frac{1}{\left\langle\xi^{3}\right\rangle} \frac{\left\langle\Delta^{2}\right\rangle_{c}}{\langle\Delta\rangle_{c}^{2}} .
$$

For a monodisperse packing the factor $g_{p}$ simplifies to 1 . In the general polydisperse case, the evaluation of $g_{p}$ necessitates an integration over the normalized particle size distribution $h(\xi)$ using the $p d f$ s of the normalized average normal force $\phi(\xi)$ acting on particles of radius $\xi$ :

$$
g_{p}=\frac{1}{\left\langle\xi^{3}\right\rangle} \int_{0}^{\infty} \xi \phi(\xi) h(\xi) \mathrm{d} \xi,
$$

as discussed in more detail in Ref. [22]. On the other hand, the nonlinear factor $b$ involves the second moment of the normalized normal force distribution function $\left\langle\Delta^{2}\right\rangle_{c} /\langle\Delta\rangle_{c}^{2}$.

Now we turn our attention to the remaining variable in Eq. (13), i.e., the normalized average overlap $\langle\Delta\rangle_{c}$. We relate it to the volumetric strain under the simplifying assumption of uniform deformation in the packing (non-affine deformations are relevant but go beyond the scope of this study). Given the displacement gradient, $u_{i, j}$, the change of the branch vector of a contact is:

$$
\mathrm{d} l_{i}=u_{i, j} l_{j},
$$


where summation is implied over repeating indices and the comma indicates the derivative with respect to the following index, i.e., the $j$-coordinate. The scalar product with the contact normal corresponds to the change of overlap $\delta$ and we assume that for small overlaps the length of the branch vector is equal to $\langle r\rangle$, so that:

$$
\mathrm{d} \delta=n_{i} \mathrm{~d} l_{i}=\langle r\rangle n_{i} u_{i, j} n_{j}
$$

For an isotropic deformation and contact distribution, as considered in this study, the off-diagonal (i.e., the deviatoric as well as the anti-symmetric) elements of the displacement gradient will cancel in average. Hence, recalling the definition of the normalized contact overlap, $\Delta_{c}=\delta_{c} /\langle r\rangle$, one can write:

$$
\mathrm{d}\langle\Delta\rangle_{c}=D \epsilon_{\mathrm{v}} .
$$

where $\epsilon_{\mathrm{v}}=\epsilon_{i i}$ is the trace of the infinitesimal strain tensor defined by $\epsilon_{i j}=\frac{1}{2}\left(u_{i, j}+u_{j, i}\right)$ and $D$ is a proportionality constant that depends on the size distribution and reflects the non-affinities in the deformation, however, this issue is beyond the scope of this study.

The average normalized overlap $\langle\Delta\rangle_{c}$ can be obtained by integrating Eq. (17), where the integral of $\epsilon_{\mathrm{v}}$, denoted by $\varepsilon_{\mathrm{v}}$, is the true or logarithmic volume change of the system, relative to the reference volume $V_{0}$, with corresponding reference volume fraction, $\nu_{0}$, which we choose - without loss of generality - to be equal to the critical, jamming volume fraction $\nu_{0}=\nu_{c}$, so that.

$$
\langle\Delta\rangle_{c}=D \int_{V_{0}}^{V} \epsilon_{\mathrm{v}}=D \varepsilon_{\mathrm{v}}=D \ln \left(\frac{\nu_{c}}{\nu}\right) .
$$

Substituting Eq. (18) into Eq. (13) we obtain for the non-dimensional pressure:

$$
p=p_{0} \frac{\nu C}{\nu_{c}}\left(-\varepsilon_{\mathrm{v}}\right)\left[1-\gamma_{p}\left(-\varepsilon_{\mathrm{v}}\right)\right],
$$

where the prefactors are condensed into $p_{0} \equiv \nu_{c} g_{p} D / 2 \pi$ and $\gamma_{p} \equiv b D / 2 g_{p}$. The implications of this, e.g., the combination $g_{p} D$ should not depend on $\nu_{c}$, will be further studied and discussed elsewhere [22].

Note that in our sign-convention, compressive strains are negative - corresponding to decreasing volume with ongoing compression - so that, accordingly, compressive stresses should be negative too. However, we rather use positive compressive stress as above, for the sake of continuity.
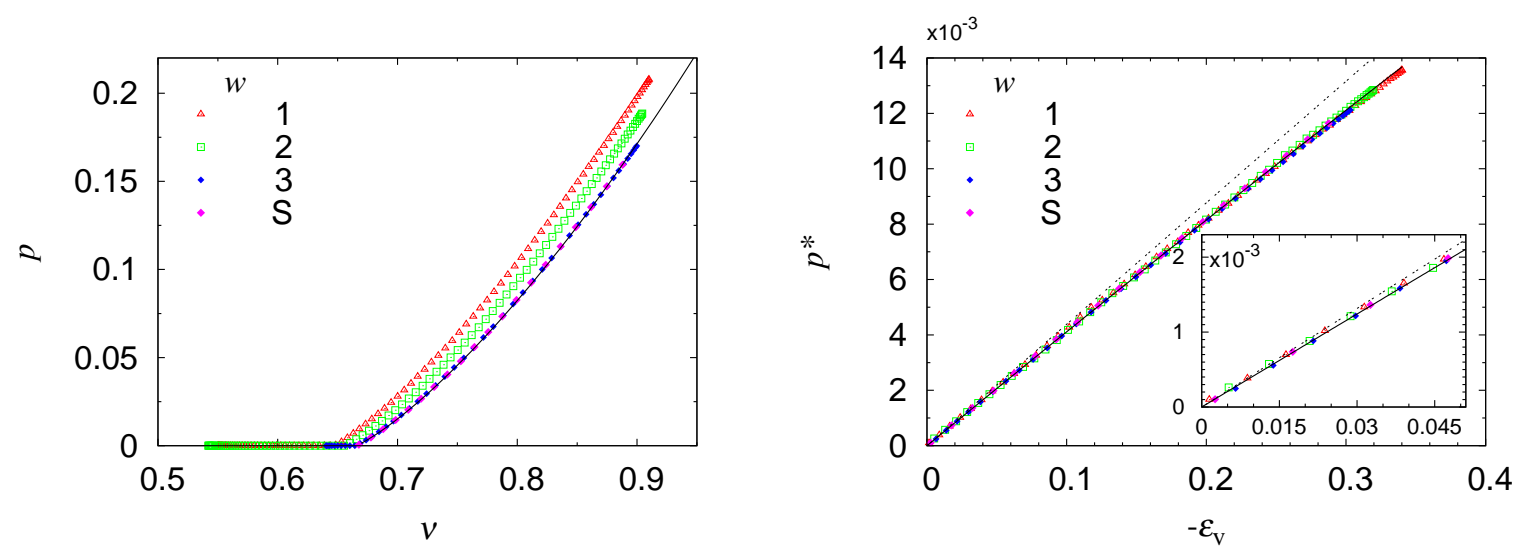

Figure 10. The dimensionless pressure as function of the volume fraction (left) (where the solid line is Eq. (19), with $\nu_{c}=0.666$ and otherwise using the numbers giving in table 3 that fit well data-set $\mathrm{S}$ with $N \approx 5000$ particles and $w=3$.) and the scaled pressure as function of the (negative) volumetric strain (right). The solid line is obtained from Eq. (20) and the dashed line is the linear approximation. Inset: Zoom into the small deformation regime. 
Figure 10 shows the non-dimensional pressure as function of volumetric strain, from representative simulations of isotropic deformation for different size distributions. Various other data (not shown, except for one that is indicated by S) using different system sizes and deformation protocols collapse with the same curves - as long as the rate of deformation is small. Interestingly, the scaled pressure

$$
p^{*}=\frac{p \nu_{c}}{\nu C}=p_{0}\left(-\varepsilon_{\mathrm{v}}\right)\left[1-\gamma_{p}\left(-\varepsilon_{\mathrm{v}}\right)\right]
$$

is independent of the polydispersity and is well represented by the linear relation in Eq. (19), namely $p^{*} \approx-p_{0} \varepsilon_{\mathrm{v}}$, valid for small deformations. The correction factor $\left[1+\gamma_{p} \varepsilon_{\mathrm{v}}\right]$ is only required for large volumetric strain. The (positive) coefficients $p_{0} \approx 0.0418$ and $\gamma_{p} \approx 0.110$ fit our data well ${ }^{3}$.

Eq. (19) now represents the constitutive relation for pressure, from which we can compute, e.g., the bulk modulus of a polydisperse packing, using the definition $B=-V(\partial p / \partial V)=\partial p / \partial\left(-\varepsilon_{\mathrm{v}}\right)=\nu \partial p / \partial \nu$. Given the dimensionless bulk modulus,

$$
B=\frac{\partial p}{\partial\left(-\varepsilon_{\mathrm{v}}\right)}=\frac{p_{0} F_{V}}{g_{3} \nu_{c}}\left[1-2 \gamma_{p}\left(-\varepsilon_{\mathrm{v}}\right)+\left(-\varepsilon_{\mathrm{v}}\right)\left[1-\gamma_{p}\left(-\varepsilon_{\mathrm{v}}\right)\right] \frac{\partial \ln \left(F_{V}\right)}{\partial\left(-\varepsilon_{\mathrm{v}}\right)}\right]
$$

with $F_{V}=\operatorname{tr}(\mathbf{F})=g_{3} \nu C$, one has an incremental evolution equation for the dimensionless stress:

$$
\mathrm{d} p=B\left(-\mathrm{d} \varepsilon_{\mathrm{v}}\right),
$$

with the incremental evolution equation for the isotropic fabric:

$$
\mathrm{d} F_{V}=F_{V}\left(1+\nu \frac{\partial C}{\partial \nu}\right)\left(-\mathrm{d} \varepsilon_{\mathrm{v}}\right),
$$

where the classical coordination number, $C=\left(1-\phi_{r}(\nu)\right) C^{*}(\nu)$, is an analytically known function of $\nu$, see Eqs. (3) and (4), involving the parameters/coefficients as summarized in table 3.

Note that the above evolution equation for the dimensionless pressure Eq. (22), together with Eqs. (21), (23) and Eqs. (3), (4), represents the main result of this study that can be easily translated into dimensional pressure and bulk modulus by multiplication with the factor $k_{n} /(2\langle r\rangle)$. As final remark, the bulk modulus does not explicitly depend on pressure, but $F_{V}$ does implicitly, hiding the pressure depence of $B$. Furthermore, the last term in the bulk modulus involves the derivative $\partial C / \partial \nu$, which can be very large close the the critical density, due to the power $\alpha<1$, and thus is not negligible. Future work should focus on the validation and comparison of the present approach with experimental data, e.g., concerning the density dependence of pressure and the pressure dependence of $B$.

\section{Summary and Conclusion}

The transition between fluid- and solid-like phases in idealized, frictionless packings of polydisperse spheres has been investigated by means of discrete element simulations of isotropic compression and decompression. As main result, an incremental constitutive relation is given in Eq. (22) for the pressure

3. The best fit quality (error less than one per-cent for all densities) is obtained when Eq. (20) is used to fit the pressure, disregarding the data very close to jamming, i.e., for the best fits, data for $\nu<\nu_{c}+0.002$ are neglected, since those are hampered by dynamic effects and are thus most unreliable - even when following a very slow unloading procedure (data-set $\mathrm{S}$ ). Thus we cannot exclude the possibility that the behavior very close to jamming turns out to be different from our results. However, as compared to the very wide range of densities covered, this concerns only a very small regime at very low pressures. The parameter $p_{0}$ is of major importance, while $\gamma_{p}$ depends on $p_{0}$ rather strongly, however, contributing only a small variation to the pressure. Furthermore, fitting power laws proportional to $\left(\nu-\nu_{c}\right)^{\beta}$ to the pressure was not possible over the whole range. For the ranges $0.67<\nu<0.72$ and $0.7<\nu<0.9$ rather good fits lead to power $\beta=1.21$ and 1.34 , respectively. 
change under isotropic deformation, to be used together with Eqs. (21), (23) and Eqs. (3), (4). The pressure evolution equation should be (i) valid for a road range of volume fractions $\nu \geq \nu_{c}$, (ii) should be rather insensitive to (moderate) polydispersity and (iii) involves only analytically known functions of the volume fraction.

The coordination number, i.e., the average number of contacts per all particles, $C$, is analyzed as function of the volume fraction in order to characterize the state of the granular packing. When the rattlers (i.e. particles with less than four contacts) are disregarded, one obtains the corrected coordination number $C^{*} \approx C /\left(1-\phi_{r}\right)$. The fraction of rattlers, $\phi_{r}$, jumps at the jamming volume fraction from $\phi_{r}=1$ to $\phi_{c}$ and then decays exponentially with increasing volume fraction. Previous studies have shown that the coordination $C^{*}$ number is discontinuous at the transition and evolves as a power law in the jammed phase close to the critical volume fraction. However, to the authors knowledge, the validity of the power law has not been checked in a broader range up to much higher volume fractions. We fitted an analytical expression of the power law to the simulation data obtained from various packings and confirm that it is not only valid in the neighborhood of $\nu_{c}$ but also for very dense packings.

The effect of different system and simulation parameters on the coordination number and the critical volume fraction have been analyzed. We find that changing the polydispersity of the packing causes a shift in the critical volume fraction, i.e., more heterogeneous packings jam at higher volume fractions. However, the power law behavior of the coordination number is not affected by polydispersity. Lowering the deformation rate has the effect of steepening the slope of the coordination number vs. volume fraction curve at the transition, which suggests that the discontinuity will be only achieved in the limit of quasistatic deformation. A study of the effect of deformation rate on the critical volume fraction based on the fit of the power law is unreliable because of the singularity at this point. We recommend that the fit should be used in conjunction with one of the methods proposed in Ref. [14] to determine $\nu_{c}$ self-consistently. Finally, we note that varying the deformation rate as well as the system size and deformation history does not have a significant effect on the evolution of the coordination number at high volume fractions: when the rattlers are removed, the power law behavior remains unaffected, at higher densities.

The structure of the contact network plays an important role in determining the mechanical properties of granular materials. In section 4 we reviewed previous theoretical predictions regarding the trace of the fabric tensor and compared them with our numerical results. The contact number density $\nu C$ obtained from the simulations and corrected by the factor $g_{3}$, which only depends on the moments of the particle size distribution, as proposed in Ref. [22], is in good agreement with the trace of the fabric tensor, so that $\operatorname{tr}(\mathbf{F})=g_{3} \nu C^{*}\left(1-\phi_{r}\right)$.

Additionaly, an incremental expression of the pressure has been derived in section 5 based on the micromechanical properties of the particles. The volumetric strain applied to the packing and the isotropic fabric was related to it, thereby enabling us to give an analytical expression for the bulk modulus that includes an evolution term of the isotropic fabric, as specified above. Scaling is observed between the numerical results for different polydispersities when the scaled pressure $p^{*}$ is plotted against volumetric strain relative to the critical configuration at volume fraction $\nu=\nu_{c}$. We note that the analytical form of the pressure does not explicitly contain a closed power-law relation. The pressure is proportional to the trace of fabric (which contains the power-law relation for the coordination number) and otherwise linear with volumetric strain - involving a rather small quadratic correction for very large strains.

In this paper we only considered isotropic deformations applied to frictionless packings of spheres. The natural next steps are to also apply deviatoric (or shear) strain and to include friction and other material parameters. The former will lead to structural anisotropy, while the latter allows to study the effect of various contact properties - like friction - on the evolution of the fabric and the stress. The evolution of, not only, pressure but also of deviatoric stresses is related to the anisotropy of the structure, see the $2 \mathrm{D}$ observations in Refs. [28, 29] and the more recent results in $3 \mathrm{D},[30,31]$, which also confirm that the scaling relation of the fabric - as observed here without friction - holds also in the presence of friction 
$[23,32]$.

We note that the jamming volume fraction $\nu_{c}$ (e.g. under cyclic loading) is not a constant, but depends on the history of the packing. This issue was not addressed in this study, but will be subject to future research.

Finally, the relations proposed in this study should be compared to experimental data in order to test their predictive value. For example, the pressure dependence of the bulk-modulus is a measurable bulk property, whereas the fraction of rattlers and the isotropic fabric are usually not easily available experimentally.

\section{Acknowledgements}

Financial support from the Delft Platform for Computational Science and Engineering is gratefully acknowledged as well as support from the research programme of the "Stichting voor Fundamenteel Onderzoek der Materie (FOM)", which is financially supported by the "Nederlandse Organisatie voor Wetenschappelijk Onderzoek (NWO)" (project number 03PGM15). We thank N. P. Kruyt and K. Bertoldi for helpful comments and discussions.

\section{References}

[1] H. M. Jaeger, S. R. Nagel, and R. P. Behringer, "Granular solids, liquids, and gases," Rev. Mod. Phys., vol. 68, no. 4, pp. 1259-1273, 1996.

[2] P. G. de Gennes, "Granular matter: a tentative view," Reviews of Modern Physics, vol. 71, no. 2, pp. 374-382, 1999.

[3] P. A. Cundall and O. D. L. Strack, "A discrete numerical model for granular assemblies," Géotechnique, vol. 29, no. 1, pp. 47-65, 1979.

[4] S. Luding, "Cohesive, frictional powders: contact models for tension," Granular matter, vol. 10, no. 4, 2008.

[5] A. J. Liu and S. R. Nagel, "Nonlinear dynamics - Jamming is not just cool any more," Nature, vol. 396, no. 6706, 1998.

[6] T. S. Majmudar, M. Sperl, S. Luding, and R. P. Behringer, "Jamming transition in granular systems," Phys. Rev. Lett., vol. 98, no. 5, p. 058001, 2007.

[7] C. S. O'Hern, S. A. Langer, A. J. Liu, and S. R. Nagel, "Random packings of frictionless particles," Phys. Rev. Lett., vol. 88, no. 7, 2002.

[8] P.-E. Peyneau and J.-N. Roux, "Solidlike behavior and anisotropy in rigid frictionless bead assemblies," Phys. Rev. E, vol. 78, no. 4, Part 1, 2008.

[9] A. Donev, S. Torquato, and F. H. Stillinger, "Pair correlation function characteristics of nearly jammed disordered and ordered hard-sphere packings," Phys. Rev. E, vol. 71, no. 1, Part 1, p. 011105, 2005.

[10] B. Scarlett, M. van der Kraan, and R. J. M. Janssen, "Porosity: a parameter with no direction," Phil. Trans. Royal Soc. London, Series A, vol. 356, no. 1747, pp. 2623-2648, 1998.

[11] A. W. Jenike, "Storage and flow of solids, bulletin no. 123," Bulletin of the University of Utah, vol. 53, no. 26, p. 198, 1964.

[12] J. Schwedes, "Review on testers for measuring flow properties of bulk solids," Granular Matter, vol. 5, no. 1, pp. 1-45, 2003. 
[13] T. S. Tan, K. K. Phoon, D. W. Hight, and Leroueil, Characterisation and engineering properties of natural soils, vol. 3-4. Taylor \& Francis, 2002.

[14] F. Göncü, O. Durán, and S. Luding, "Jamming in frictionless packings of spheres: determination of the critical volume fraction," in Powders and Grains 2009, 2009.

[15] C. S. O'Hern, L. E. Silbert, A. J. Liu, and S. R. Nagel, "Jamming at zero temperature and zero applied stress: The epitome of disorder," Phys. Rev. E, vol. 68, no. 1, Part 1, 2003.

[16] I. Agnolin and J.-N. Roux, "Internal states of model isotropic granular packings. I. Assembling process, geometry, and contact networks," Phys. Rev. E, vol. 76, DEC 2007.

[17] M. Madadi, O. Tsoungui, M. Lätzel, and S. Luding, "On the fabric tensor of polydisperse granular media in 2d," Int. J. Sol. Struct., vol. 41, no. 9-10, pp. 2563-2580, 2004.

[18] J. D. Goddard, "Continuum modeling of granular assemblies," in Physics of dry granular media NATO ASI Series E 350 (H. J. Herrmann, J. P. Hovi, and S. Luding, eds.), (Dordrecht), pp. 1-24, Kluwer Academic Publishers, 1998.

[19] M. Madadi, S. M. Peyghoon, and S. Luding, "Stress and fabric for polydisperse, frictionless, dense 2d granular media," in Powders and Grains 2005 (R. Garcia-Rojo, H. J. Herrmann, and S. McNamara, eds.), (Leiden, Netherlands), pp. 93-97, Balkema, 2005.

[20] H. N. Zhu, M. M. Mehrabadi, and M. Massoudi, "Incorporating the effects of fabric in the dilatant double shearing model for planar deformation of granular materials," Int. J. of Plasticity, vol. 22, no. $4,2006$.

[21] W. Wu, "Rational approach to anisotropy of sand," Int. J. for Numerical and Analytical Methods in Geomechanics, vol. 22, no. 11, 1998.

[22] O. Durán, M. Madadi, and S. Luding, "Fabric and stress tensors for frictionless, polydisperse, three dimensional granular media." 2010.

[23] S. Luding, "Constitutive relations for the shear band evolution in granular matter under large strain," Particuology, vol. 6, no. 6, 2008.

[24] L. E. Silbert, D. Ertas, G. S. Grest, T. C. Halsey, and D. Levine, "Geometry of frictionless and frictional sphere packings," Phys. Rev. E, vol. 65, p. 051302, MAR 2002.

[25] N. P. Kruyt, "Three-dimensional lattice-based dispersion relations for granular materials," in IUTAM-ISIMM Symposium on Mathematical Modeling and Physical Instances of Granular Flows (J. Goddard, P. Giovine, and J. T. Jenkins, eds.), (Reggio Calabria (Italy), 14.18 September 2009), pp. 405-415, AIP, 2010.

[26] C. T. David, R. G. Rojo, H. J. Herrmann, and S. Luding, "Hysteresis and creep in powders and grains," in Powders and Grains 2005 (R. Garcia-Rojo, H. J. Herrmann, and S. McNamara, eds.), (Leiden, Netherlands), pp. 291-294, Balkema, 2005.

[27] M. Lätzel, S. Luding, and H. J. Herrmann, "Macroscopic material properties from quasi-static, microscopic simulations of a two-dimensional shear-cell," Granular Matter, vol. 2, no. 3, pp. 123$135,2000$.

[28] S. Luding, "Micro-macro models for anisotropic granular media," in Modelling of Cohesive-Frictional Materials (P. A. Vermeer, W. Ehlers, H. J. Herrmann, and E. Ramm, eds.), (Leiden, Netherlands), pp. 195-206, Balkema, 2004.

[29] S. Luding, "Anisotropy in cohesive, frictional granular media," J. Phys.: Condens. Matter, vol. 17, pp. S2623-S2640, 2005.

[30] O. Duran, N. P. Kruyt, and S. Luding, "Analysis of three-dimensional micro-mechanical strain formulations for granular materials: evaluation of accuracy," Int. J. of Solids and Structures, vol. 47, no. , pp. 251-260, 2010.

[31] O. Duran, N. P. Kruyt, and S. Luding, "Micro-mechanical analysis of deformation characteristics of three-dimensional granular materials," Int. J. of Solids and Structures, vol., no. , p. in press, 2010.

[32] S. Luding, "The effect of friction on wide shear bands," Particulate Science and Technology, vol. 26, 
no. 1, 2008 . 
(a)

\begin{tabular}{cccc}
\hline$w$ & 1 & 2 & 3 \\
\hline$C_{0}$ & 6.0000 & 5.9690 & 6.1158 \\
$C_{1}$ & 8.7989 & 8.5539 & 7.9439 \\
$\alpha$ & 0.5363 & 0.5776 & 0.5737 \\
$\nu_{c}$ & 0.6524 & 0.6582 & 0.6718 \\
\hline
\end{tabular}

(b)

\begin{tabular}{cccc}
\hline$w$ & 1 & 2 & 3 \\
\hline$C_{0}$ & 6 & 6 & 6 \\
$C_{1}$ & 8.7363 & 8.5561 & 7.9367 \\
$\alpha$ & 0.5662 & 0.5826 & 0.5542 \\
$\nu_{c}$ & 0.6548 & 0.6585 & 0.6707 \\
\hline
\end{tabular}

Table 1

(a) Numerical values of the fit-parameters obtained by fitting Eq. (4) to the un-loading simulation data of Fig. 4, in the intervals [0.655:0.85], [0.66:0.85] and [0.672:0.85] for $w=1,2$ and 3, respectively. (b) Numerical values of the fit-parameters obtained by fitting Eq. (4) to the un-loading simulation data of Fig. 4 , in the same intervals and fixing $C_{0}=6$. 


\begin{tabular}{|c|c|c|c|c|c|c|c|c|c|}
\hline & \multicolumn{2}{|c|}{$N=1000$} & \multicolumn{2}{|c|}{$N=5000$} & \multicolumn{5}{|c|}{$N=10000$} \\
\hline & $D=1$ & $D=0.5$ & $D=1$ & $D=0.5$ & $D=1$ & $D=0.5$ & $D=0.1$ & $D=0.01$ & $D=0.001$ \\
\hline$C_{0}$ & 5.0256 & 5.8221 & 5.7645 & 5.8838 & 5.7645 & 5.7887 & 6.0643 & 6.1587 & 6.1853 \\
\hline$C_{1}$ & 7.5938 & 8.4875 & 8.2019 & 8.1661 & 8.2019 & 7.9915 & 8.4204 & 8.8347 & 8.7514 \\
\hline$\alpha$ & 0.3904 & 0.5572 & 0.5279 & 0.5431 & 0.5279 & 0.5199 & 0.5909 & 0.6301 & 0.6318 \\
\hline$\nu_{c}$ & 0.6650 & 0.6650 & 0.6654 & 0.6647 & 0.6654 & 0.6652 & 0.6648 & 0.6645 & 0.6644 \\
\hline$\nu_{c}^{\dagger}$ & 0.6652 & 0.6644 & 0.6624 & 0.6620 & 0.6627 & 0.6632 & 0.6633 & 0.6634 & 0.6633 \\
\hline
\end{tabular}

Table 2

Numerical values of the fit parameters of Eq. (4) for various system sizes and loading rates. All packings have the polydispersity parameter $w=3$ and are deformed within the range $\nu: 0.64 \rightleftarrows 0.75$. The fits are performed in the intervals $\left[\nu_{1}: \nu_{2}\right]$, with $\nu_{1}=0.665$ and $\nu_{2}=0.75 . \nu_{c}^{\dagger}$ are the volume fractions at which the pressure vanishes during unloading, see Ref. [14]. Note that the data in table 1 are slightly different (since they come from simulations with different initial conditions), which tells us something about the sensitivity and variation of parameters with different initial configurations.

Table 3

\begin{tabular}{|c|c|c|c|}
\hline \multicolumn{4}{|l|}{ fit parameters for $C(\nu)$} \\
\hline jamming volume fraction & $\nu_{c}$ & $0.66 \pm 0.01$ & variable $\nu_{c}(D, w, \ldots)$ \\
\hline coordination number at jamming & $C_{0}$ & 6 & exact \\
\hline prefactor for the algebraic coordination number & $C_{1}$ & $8 \pm 0.5$ & variable \\
\hline power for the algebraic coordination number & $\alpha$ & $0.58 \pm 0.05$ & approximate \\
\hline \multicolumn{4}{|l|}{ fit parameters for $\phi_{r}(\nu)$} \\
\hline fraction of rattlers at jamming & $\phi_{c}$ & $0.13 \pm 0.03$ & approximate \\
\hline decay rate of fraction of rattlers & $\phi_{\nu}$ & $15 \pm 2$ & approximate \\
\hline \multicolumn{4}{|l|}{ relation between fabric and contact number density } \\
\hline polydispersity correction factor & $g_{3}$ & $\geq 1$ & variable $g_{3}(w)$ \\
\hline \multicolumn{4}{|l|}{ fit parameters for $p$} \\
\hline linear pressure factor & $p_{0}$ & $0.0418 \pm 0.001$ & approximate \\
\hline non-linear pressure factor & $\gamma_{p}$ & 0.110 & strongly dependent on $p_{0}$ \\
\hline
\end{tabular}

Summary of the coefficients involved in the constitutive relations for the pressure $p$ and the isotropic fabric $F_{V}$. In the column right of the symbols are given typical values - some of them are exact, some are fits with a broad spread and some are not changing so much. In the last column some strong dependencies are indicated, e.g., $g_{3}$ depends only on the width of the size distribution, $w$, but not on other variables. 